\title{
A Precession Electron Diffraction and EELS Study of Beta-phase Evolution in Nano-crystalline Mg-9 wt. \% Al Thin Films during Heat Treatment
}

\author{
Karen Kruska ${ }^{1}$, Daniel J Edwards ${ }^{1}$, Rama S Vemuri ${ }^{1}$, Libor Kovarik ${ }^{1}$, Aashish Rohatgi ${ }^{1}$, Nigel D \\ Browning ${ }^{1}$
}

${ }^{1}$ Pacific Northwest National Laboratory, \{FCSD, EED, EMSL\}, Richland, WA, United States.

Modern transmission electron microscopy (TEM) and spectroscopy techniques routinely probe the microstructure and local compositions in nanocrystalline materials using various analytical techniques such as electron energy-loss spectroscopy (EELS) or energy dispersive x-ray spectroscopy (EDX) maps. Electron diffraction is another technique the can help to identify phases in materials, but in applying such a technique, it is cumbersome to obtain statistically relevant information from nanomaterials. With the development of precession electron diffraction (PED) mapping and the use of image correlation for pattern identification, novel information can be obtained relatively rapidly that covers a much larger number of grains [1]. Combined with elemental mapping, this provides a powerful technique to characterize the distribution of phases and chemical distribution in nanomaterials.

The goal of a broader study at PNNL is to understand the evolution of $\mathrm{Mg}_{17} \mathrm{Al}_{12} \beta$-phase precipitates as a function of heat-treatment in an automotive Mg AZ91 (Mg-9 wt.\% Al- 1 wt.\% Zn) alloy. In this work, EELS and PED on a cold-FEG JEOL ARM200CF TEM were used to investigate the evolution of the $\beta$ phase precipitates in a binary Mg-9 wt.\% Al thin film subjected to a heat treatment at 300C for $30 \mathrm{~min}$. $80 \mathrm{~nm}$ Mg-9Al thin films were deposited on a $25 \mathrm{~nm}$ amorphous $\mathrm{Si}_{3} \mathrm{~N}_{4}$ support membrane. Heating was conducted in a Gatan single-tilt heating stage. EELS acquisition was performed with a convergence halfangle of 34.4 mrad and a collection half-angle of 82.6 mrad with a dispersion of $0.25 \mathrm{eV}$. Multivariate statistical analysis (MSA) was used to reduce statistical noise in EELS data [2]. PED data was collected in nanoprobe mode using a probe size of $1.2 \mathrm{~nm}$ at $50 \mathrm{~Hz}$, a precession angle of 0.69 deg and a step size of 4 to $5 \mathrm{~nm}$.

The PED data in Figure 1 shows the $\alpha-\mathrm{Mg}-\mathrm{Al}$ (red), and the $\beta-\mathrm{Mg}_{17} \mathrm{Al}_{12}$ (green) that nucleated and grew upon heating from an as-sputtered $\alpha$-Mg-9 wt.\% Al film. The black pixels in Figures 1a and b show pixels of high uncertainty [1]. Much higher reliability could be achieved when precession was enabled during mapping. The apparent area fraction of the $\beta$-phase in Figure $1 \mathrm{~b}$ is $\sim 8 \%$. In comparison, the EELS relative composition maps in Figures 2b and c show 26 \% "Al-rich" grains of which majority have an apparent Al concentration between 10-30\%. The stoichiometric concentration of Al in the $\beta$ precipitates of $41 \%$ was only measured in few grains. The maximum solubility of $\mathrm{Al}$ in $\mathrm{Mg}$ is $\sim 12 \%$, and existence of grains with $12 \%<\mathrm{Al}<41 \%$ is not predicted by the equilibrium Mg-Al phase diagram. Given the moderate cooling rate after heat treatment, such non-equilibrium conditions seem unlikely. An alternative interpretation is the formation of $\beta$-precipitates that do not stretch over the entire thickness of the thin film. Thus, while PED only identifies the strongest diffraction pattern from the two overlapping phases in the beam path, the 2D EELS projection leads to a perceived Al concentration between $12 \%$ and $41 \%$. Implications of these findings for the treatment of PED data in this set-up will be discussed [3].

\section{References}

[1] E.F. Rauch, M. Véron, Materials Characterization 98 (2014), p. 1-9 
[2] G. Lucas, P. Burdet, M. Cantoni, C. Hebert, Micron 52-53 (2013), p. 49-56

[3] This work was sponsored by the Vehicle Technologies Office of the U.S. Department of Energy. The utilized PED system was transferred from Portland State University. A portion of the research was performed using EMSL, a national scientific user facility sponsored by the Department of Energy's Office of Biological and Environmental Research and located at Pacific Northwest National Laboratory.
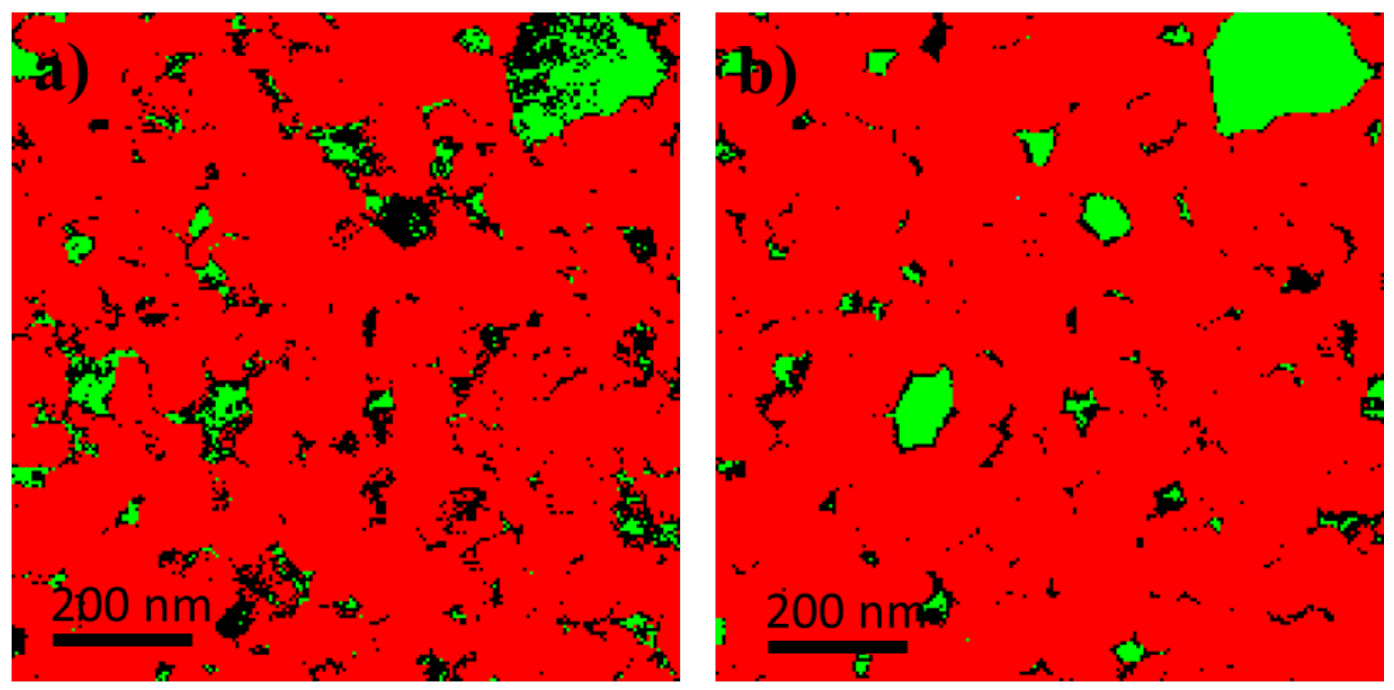

$\alpha-\mathrm{Mg}$ phase

$\beta-\mathrm{Mg}_{17} \mathrm{Al}_{12}$ phase

Figure 1: PED data showing the phases in Mg-9Al thin film. a) Phase map acquired without precession. b) Phase map acquired with precession. Black pixels indicate less than 15\% reliability in the indexing.

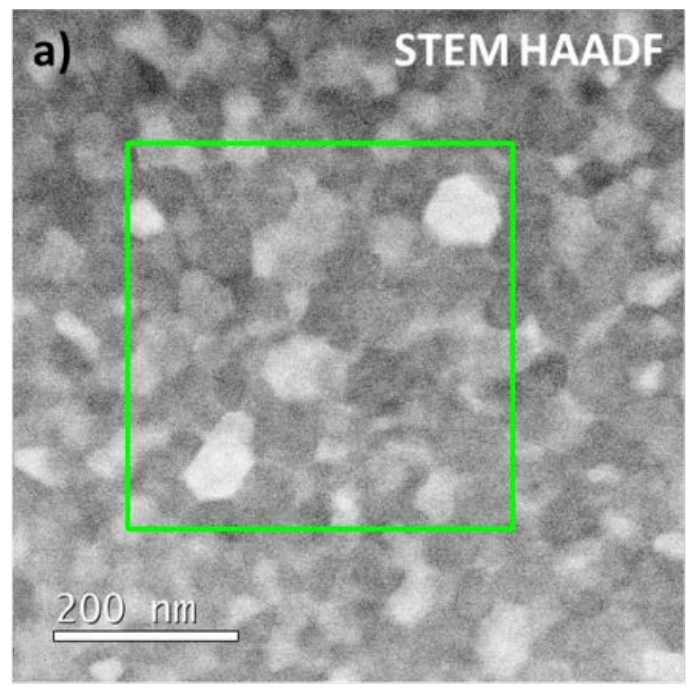

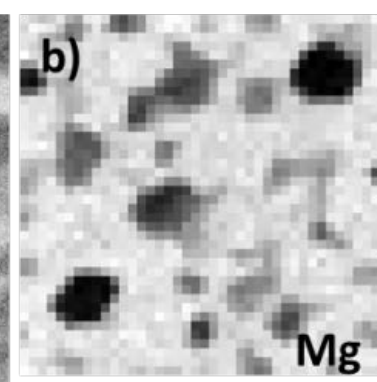

d)

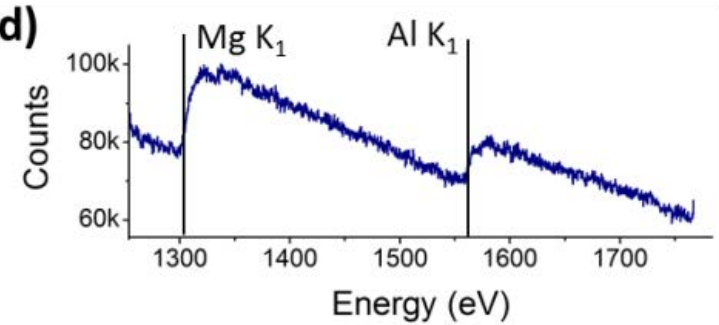

Figure2: EELS data showing the phases in Mg-9Al thin film. a) STEM HAADF image showing the analyzed area $b$ ) Mg relative composition map for data reconstructed with 6 Eigenvalues. $c$ ) Respective Al relative composition map. d) EELS spectrum showing the poor signal:noise during mapping. 